\title{
A novel chemosynthetic peptide with $\beta$-sheet motif efficiently kills Klebsiella pneumoniae in a mouse model
}

This article was published in the following Dove Press journal:

International Journal of Nanomedicine

9 February 2015

Number of times this article has been viewed

\author{
Shirui $\operatorname{Tan}^{1,2, *}$ \\ Changpei Gan ${ }^{1,3, *}$ \\ Rongpeng $\mathrm{Li}^{\prime}$ \\ Yan Ye' \\ Shuang Zhang',3 \\ XuWu' \\ Yi Yan Yang ${ }^{4}$ \\ Weimin Fan $^{5}$ \\ Min Wu'
}

'Department of Basic Sciences, School of Medicine and Health Sciences University of North Dakota, Grand Forks, ND, USA; ${ }^{2}$ Laboratory of Biochemistry and Molecular Biology, School of Life Sciences, Yunnan University, Kunming, People's Republic of China; ${ }^{3}$ State Key Laboratory of Biotherapy, West China Hospital, Sichuan University, Chengdu, People's Republic of China; ${ }^{4}$ Institute of Bioengineering and Nanotechnology, The Nanos, Singapore; ${ }^{5}$ Program of Innovative Cancer Therapeutics, First Affiliated Hospital of Zhejiang University College of Medicine, Hangzhou, People's Republic of China

*These authors contributed equally to this work
Correspondence: Yi Yan Yang; Min Wu Department of Basic Sciences, School of Medicine and Health Sciences, University of North Dakota, Grand Forks, ND 58203-9037, USA

Tel +70I 7774875

Fax +70I 7772382

Email yyyang@ibn.a-star.edu.sg; min.wu@med.und.edu
Abstract: Klebsiella pneumoniae $(K p)$ is one of the most common pathogens in nosocomial infections and is increasingly becoming multiple drug resistant. However, the molecular pathogenesis of $K p$ in causing tissue injury and dysregulated host defense remains elusive, further dampening the development of novel therapeutic measures. We have previously screened a series of synthetic antimicrobial beta-sheet forming peptides and identified a peptide (IRIKIRIK; ie, IK8L) with a broad range of bactericidal activity and low cytotoxicity in vitro. Here, employing an animal model, we investigated the antibacterial effects of IK8L in acute infection and demonstrated that peritoneal injection of IK8L to mice down-regulated inflammatory cytokines, alleviated lung injury, and importantly, decreased mortality compared to sham-injected controls. In addition, a math model was used to evaluate in vivo imaging data and predict infection progression in infected live animals. Mechanistically, IK8L can kill $K p$ by inhibiting biofilm formation and modulating production of inflammatory cytokines through the STAT3/ JAK signaling both in vitro and in vivo. Collectively, these findings reveal that IK8L may have potential for preventing or treating $K p$ infection.

Keywords: bacterial pathogenesis, inflammatory cytokines, infectious diseases, bactericidal activity, antimicrobial peptides, STAT3/JAK signaling transduction

\section{Introduction}

Frequent use of antibiotics in the agricultural and biomedical fields has caused a surge in antibiotic-resistant pathogen strains. ${ }^{1}$ Infection by multidrug resistant (MDR) bacteria, such as Klebsiella pneumoniae $(K p)$, is challenging to treat, ${ }^{2,3}$ thereby compounding existing conditions ${ }^{4}$ such as asthma, cystic fibrosis, and chronic obstructive pulmonary disease (COPD).$^{5-7}$ In the United States, COPD alone causes more than 157,000 deaths every year. ${ }^{8} \mathrm{Kp}$ is the third most isolated microorganism in blood cultures from patients with various complications. ${ }^{9}$ Therefore, understanding the mechanism of $K p$ antibiotic resistance is becoming an important topic in human health, which may provide insight into therapeutic strategies to control $K p$ infection.

To effectively eradicate antibiotic-resistant pathogens from afflicted hosts, many new antibacterial agents have been developed, including ertapenem, ${ }^{10}$ levaquin, and meropenem. ${ }^{11}$ Previously, antimicrobial peptides (AMPs) having cationic charge and amphipathic structures have been thought to be the best antibacterial agents thanks to their small size, heat stability, and broad-spectrum antibacterial activity. ${ }^{12}$ Besides, AMPs as endogenous antibiotics have extensive capabilities in regulating inflammation, wound repair, and the adaptive immune system in eukaryote host defense. ${ }^{13,14}$ However, natural AMPs possess ostensible toxicity to humans and are difficult to prepare on a large scale. ${ }^{15}$ Although improved techniques of isolation enabled larger 
quantity preparation, most of AMPs have been found to be unsuitable for clinical application due to their potential systemic toxicity. ${ }^{16}$ In treatment of $K p$ infection, the disadvantage of this natural peptide is its high systemic toxicity. Alternatively, artificially synthesized antibacterial peptides seem to be good candidates, as they have similar characteristics and efficient antibacterial ability with natural AMPs. ${ }^{17}$ However, significant toxicity to humans is still currently an unbridgeable gap to these artificially synthesized antibacterial peptides, from the laboratory to the clinic.

To overcome this limitation, the quest continues for artificially synthesized peptides to better control microorganisms and infectious diseases. We focused on developing new artificially synthesized peptides with low toxicity and high efficacy in killing pathogenic microorganisms. Having previously examined bactericidal activity in vitro, we set out to systemically evaluate the bactericidal activity of IRIKIRIK (ie, IK8L) against a clinically significant pathogen $K p$ infection in animal models.

\section{Materials and methods}

\section{Bacterial strains and culture conditions}

K. pneumoniae (Kp; American Type Culture Collection [ATCC] 43816 serotype II) was obtained from Dr Virginia Miller (University of North Carolina, Chapel Hill, NC, USA) and was used for infection of mice and cells. $K p$-GFP (green fluorescent protein) (ATCC 43816 serotype II) was kindly provided by Dr Steven Clegg (University of Iowa Carver College of Medicine, Iowa City, IA, USA). Kp Xen-39 (ATCC 93A 5370), an engineered bioluminescent pathogenic bacterium strain expressing bioluminescence, was used for imaging both in vitro and in vivo with Caliper's Xenogen IVIS XRII optical imaging technology (Caliper; PerkinElmer, Waltham, MA, USA). Bacteria were grown in Luria-Bertani (LB) broth at $37^{\circ} \mathrm{C}$ for 16 hours, followed by centrifuging at $5000 \mathrm{~g}$ for 5 minutes, and subsequently washed with sterile phosphate-buffered saline (PBS) for infection. ${ }^{18}$

\section{Mouse infection}

All animal procedures were approved by Institutional Animal Care and Use Committee at the University of North Dakota. C57BL/6J mice were purchased from Harlan Laboratory (Indianapolis, IN, USA). Mice were housed in a temperature- and humidity-controlled environment, and had free access to food and water. After anesthesia with $40 \mathrm{mg} / \mathrm{kg}$ ketamine, mice were instilled with $1 \times 10^{5}$ (four mice/group) colony-forming units (CFUs) of $K p$ by intranasal instillation, and sacrificed when they were moribund. ${ }^{19,20}$ Survival was determined using Kaplan-Meier curve. After bronchoalveolar lavage (BAL), the lung was excised for homogenization or fixed in $10 \%$ formalin.

\section{Cell estimation in BAL and isolation of alveolar macrophages (AMs)}

BAL was performed five times for each $1.0 \mathrm{~mL}$ (except the first $0.6 \mathrm{~mL}$ ) of lavage fluid; a cell smear was made from the BAL fluid for cell differential counting. AMs were collected from the BAL pellet after centrifuging at 2,000 $\times g$ for 5 minutes at $4^{\circ} \mathrm{C}$ and cultivated in RPMI (Roswell Park Memorial Institute) 1640 medium supplemented with $10 \%$ newborn calf serum and penicillin/ streptomycin in a $5 \% \mathrm{CO}_{2}$ incubator.

\section{Peptide}

IK8L was identified and described. ${ }^{21}$ The peptide was synthesized by GL Biochem (Shanghai, People's Republic of China) and purified to greater than $95 \%$ through analytical reverse phase high-performance liquid chromatography.

\section{In vivo imaging}

Mice were infected with bioluminescence-emitting $K p$ (Xen-39, $1 \times 10^{5} \mathrm{CFU}$ ) following anesthesia by ketamine. At various time points postinfection, whole body imaging of the infected mouse was obtained under an IVIS XRII system. Additionally, the distribution of $K p$ in various organs was semiquantitatively analyzed through the calculated intensity of bioluminescence and also confirmed by direct measurement of CFUs in tissues recovered after imaging. ${ }^{22}$

\section{IK8L treatment}

Mice were randomly divided into a control group $(n=4)$ and an IK8L-treated group $(n=4)$. The IK8L-treated group received IK8L suspension ( $20 \mathrm{mg} / \mathrm{kg}$ body weight) through tail vein injection. After 4 hours, mice were infected with $K p$ by intranasal instillation.

\section{Biofilm assays}

\section{Biofilm formation assay in test tube}

Biofilm formation in test tubes was measured according to the method that O'Toole described previously. ${ }^{23}$ Briefly, test tubes were inoculated from overnight LB-grown bacterial cultures containing IK8L. After that, the cells were grown for 24 hours at $37^{\circ} \mathrm{C}$; then the test tubes were stained with crystal violet and quantified by dissolution using 30\% acetic acid and measured at an absorbance at $550 \mathrm{~nm}$. 


\section{CFU of biofilms}

Quantification of biofilm bacteria with or without IK8L treatment was performed as follows. The wells were washed six times with saline in order to remove any planktonic cells, $100 \mu \mathrm{L}$ of saline was added to each well, and the samples were independently sonicated for 8 seconds by using a VC505 sonicator (Sonics and Materials, Inc, Newtown, CT, USA), followed by plating on agar dishes for 18 hours at $37^{\circ} \mathrm{C}$ to obtain the $\mathrm{CFU}$ counts.

\section{Cell infection experiments}

After cultivating in LB broth at $37^{\circ} \mathrm{C}$ with vigorous shaking overnight, $K p$ was centrifuged at $6,000 \times g$ for 5 minutes, then resuspended in $5 \mathrm{~mL}$ fresh $\mathrm{LB}$ broth to grow until the midlogarithmic phase. The concentration of bacteria was estimated by reading optical density (OD) at $600 \mathrm{~nm}\left(0.1 \mathrm{OD}=1 \times 10^{8}\right.$ cells $/ \mathrm{mL}$ ). Before infection, cells were washed once with PBS, and replaced with both serum and antibiotic-free medium immediately. Macrophage cells were infected by $K p$ at the multiplicity of infection (MOI) of 10:1 bacteria-cell ratio for 1 hour and then washed three times with PBS to remove the free bacteria. Bacteria on the surface of the cells were killed by adding $100 \mu \mathrm{g} / \mathrm{mL}$ of polymyxin B and incubated for another 1 hour. The amount of the intracellular bacteria was determined by CFU counts. ${ }^{22,24}$

\section{Cell culture}

AMs were isolated from the bronchoalveolar lavage fluid (BALF). By centrifuging at $2000 \mathrm{rpm}$, the pellet was resuspended and cultured in RPMI 1640 medium containing $10 \%$ newborn bovine serum (HyClone Laboratories, Logan, UT, USA) and $100 \mathrm{U} / \mathrm{mL}$ of penicillin/streptomycin (Life Technologies, Rockville, MD, USA) in a $37^{\circ} \mathrm{C}$ incubator with $5 \% \mathrm{CO}_{2}$. Mouse alveolar macrophage cell line (MH-S) was obtained from American Type Culture Collection (ATCC, Manassas, VA, USA) and maintained following the manufacturer's instructions.

\section{3-(4,5-dimethyl-2-thiazolyl)-2,5- diphenyltetrazolium bromide assay}

This assay measures color change of 3-(4,5-dimethyl-2thiazolyl)-2,5-diphenyltetrazolium bromide (MTT) upon reduction by enzymes to assess the viability of AM. AM cells were obtained from infected mice and processed as above. Then, $1 \mu \mathrm{g} / \mathrm{mL}$ MTT dye (final concentration) was added to each well. The cells were incubated at $37^{\circ} \mathrm{C}$ until the color change occurred. The dye was reduced to form a purple formazan product inside living cells. Stop solution (10\% dimethyl sulfoxide [DMSO]; $10 \%$ sodium dodecyl sulfate (SDS) in $50 \mathrm{mM}$ HEPES (2-[4-(2-Hydroxyethyl)- 1-piperazinyl]ethanesulfonic acid) buffer) was added to dissolve the formazan product, and the absorbance was quantified by measuring a wavelength of $560 \mathrm{~nm}$ using a spectrometer plate reader. ${ }^{25}$

\section{Nitroblue tetrazolium assay}

This assay was used to determine the production of superoxide anion in AM cells. AM cells from BAL were grown in a 96-well plate in serum-containing medium at $37^{\circ} \mathrm{C}$ for 4 hours. We added $1 \mu \mathrm{g} / \mathrm{mL}$ nitroblue tetrazolium (NBT) dye (Sigma, St Louis, MO, USA) to each well. The cells were incubated at $37^{\circ} \mathrm{C}$ for 1 hour or until color developed. The dye is yellow and gives a blue color formazan product upon reduction by superoxide. The reaction was terminated by adding $100 \mu \mathrm{L}$ of stop solution (10\% DMSO; $10 \%$ SDS in $50 \mathrm{mM}$ HEPES buffer). The plate was left at room temperature overnight for complete dissolution of formazan, and absorbance at $560 \mathrm{~nm}$ was recorded using a multiscan plate reader to quantify the concentration of superoxide anion. Triplicates were done for each sample and control. ${ }^{26}$

\section{Tissue myeloperoxidase assay}

Samples were homogenized in $50 \mathrm{mM}$ hexadecyltrimethylammonium bromide, $50 \mathrm{mM} \mathrm{KH_{2 }} \mathrm{PO} 4, \mathrm{pH} 6.0,0.5 \mathrm{mM}$ EDTA (ethylenediaminetetraacetic acid) at $1 \mathrm{~mL} / 100 \mathrm{mg}$ of tissue and centrifuged for 15 minutes at $12,000 \mathrm{rpm}$ at $4^{\circ} \mathrm{C}$. Supernatants were decanted, and $100 \mu \mathrm{L}$ of reaction

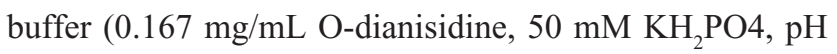
6.0, $0.0005 \% \mathrm{mM} \mathrm{H}_{2} \mathrm{O}_{2}$ ) was added to $100 \mu \mathrm{L}$ of sample. Absorbance was read at $460 \mathrm{~nm}$ at 2-minute intervals. Duplicates were done for each sample and control. ${ }^{27}$

\section{Dihydrodichlorofluorescein diacetate assay} Dihydrodichlorofluorescein diacetate $\left(\mathrm{H}_{2} \mathrm{DCF}-\mathrm{DA}\right)$ dye (Molecular Probes, Carlsbad, CA, USA) does not normally fluoresce but emits green fluorescence upon reaction with cellular superoxide. AM cells were obtained from $K p$-infected mice and an equal amount of dye $(1 \mu \mathrm{g} / \mathrm{mL})$ was added. After 1 hour incubation, fluorescence was measured by a fluorescence plate reader (BioTek, Winooski, VT, USA) using $485 \mathrm{~nm}$ excitation and a $528 \mathrm{~nm}$ emission filter. ${ }^{28}$

\section{Histological analysis}

The lung was fixed in 10\% formalin using a routine histologic procedure. Ten microlitres of BAL and blood were applied evenly on microscope slides. The formalin-fixed tissues were used for hematoxylin and eosin (H\&E) staining to examine tissue damage postinfection. After Hema staining (Thermo Fisher, 
Waltham, MA, USA), the number of polymorphonuclear neutrophils (PMN) were counted using a light microscope.

\section{Cytokine profiling}

We measured cytokine concentrations in the first $0.6 \mathrm{~mL}$ of BAL fluid (which could reflect the original levels in the lung) collected at the indicated times after infection by standard enzyme-linked immunosorbent assay (ELISA) kits following the manufacturer's instructions (eBioscience Inc., San Diego, CA, USA)..$^{18-20,29,30}$

\section{Western blotting}

Lung tissues were lysed and homogenized in a radioimmunoprecipitation assay containing a protease inhibitor cocktail (Sigma, St Louis, MO, USA). The supernatants were collected and the protein concentration was quantitated. The lysates were boiled for 10 minutes, and an equal amount of each sample was applied to $12 \%$ SDS-polyacrylamide mini gels and electrophoresed. $\beta$-actin was used as a loading control, whereas different primary antibodies were used to determine the protein levels in each sample. ${ }^{31,32}$

\section{Confocal laser scanning microscopy}

Cells were cultured in glass-bottomed dishes (MatTek, Ashland, MA, USA) for immunostaining following the manufacturer's instructions. For lipid raft staining, the cells were added with cholera toxin B chain (CTB) (red) containing fluorescein to track sphingolipid-rich lipid rafts. ${ }^{33}$ The images were obtained by LSM 510 Meta confocal microscope (Carl Zeiss Microimaging, Thornwood, NY, USA).

\section{Flow cytometry assay}

AM cells were treated with IK8L or various controls in a glass-bottomed plate for 4 hours. Apoptotic cells were stained by Annexin V-fluorescein isothiocyanate-stained cells and analyzed by flow cytometry (BD Biosciences, San Jose, CA, USA). ${ }^{34}$

\section{Statistical analysis}

Each experiment was conducted in triplicate. The differences in outcomes of the IK8L-treated mice were presented as percent or amount of change compared to the control after $K p$ infection. Data were analyzed by one-way analysis of variance (ANOVA) (Tukey's post hoc) or Mann-Whitney $U$-test using GraphPad software. ${ }^{35,36}$ The survival rate was calculated using a Kaplan-Meier curve, with $P<0.05$ being significant from a log-rank test. ${ }^{37}$

\section{Results \\ Peptide (IK8L) decreased mortality rates and infection severity of $K p$-infected mice}

We have recently reported the design of a short $\beta$-sheet forming peptide IK8L that is made of eight natural L-amino acids and demonstrates extensive and highly selective antibacterial activity against clinically isolated MDR bacteria, and its median lethal dose in mice through tail vein injection was $35.2 \mathrm{mg} / \mathrm{kg} .{ }^{38}$ To assess the physiological function of IK8L in vivo bacterial infection, IK8L was injected into C57BL/6J mice. The mice were then intranasally instilled with $K p$ Xen-39 at $1 \times 10^{5} \mathrm{CFU}$ in $50 \mu \mathrm{L}$ of PBS per mouse (four mice per group).${ }^{19}$ Although the distribution of the bacteria in the enterocelia was somewhat variable, the infected mice exhibited much wider dissemination of bioluminescence in the area of thoracic cavity 4 hours postinfection with in vivo dynamic analysis using an IVIS XRII 200 biophotonic imager. However, the dissemination areas in infected mice were more constrained and only localized in one lung in IK8L-treated mice than those of sham-treated mice (Figure 1A). In order to predict the infection level, a math model of the relative luminescence unit counts (LUC) units vs time in the Kp Xen-39 infected mice was applied to our experiment. The LUC units in the first 12 hours after the infection were collected from the IVIS XRII 200 biophotonic imager. The regression equations for sham-treated and IK8L-treated groups were

and

$$
y=92967+47002 x
$$

$$
y=52770+6546 x,
$$

respectively (Figure $\mathrm{S} 2 \mathrm{~A}$ ), where the $\mathrm{x}$ axis represents infection time and the $y$ axis represents the level of infection. These equations suggest that bacterial dissemination is dependent on the diffusing force in a constant manner. It is possible that these two equations can predict the relative LUC units over a long period of time. According to the equations, the LUC units at 24 hours, 36 hours, 48 hours, and 50 hours were predicted (Figure S2B). The results of the infected mice by $K p$ Xen- 39 of $1 \times 10^{5} \mathrm{CFU}$ showed that the actual values were highly comparable to the predicted values at each of the corresponding time points (Figure S2B). Overall, the math model used here may enable the prediction of infection levels in a long time period. Apparently, much broader infection areas were observed in control mice; approximately $50 \%$ of infected mice died within 

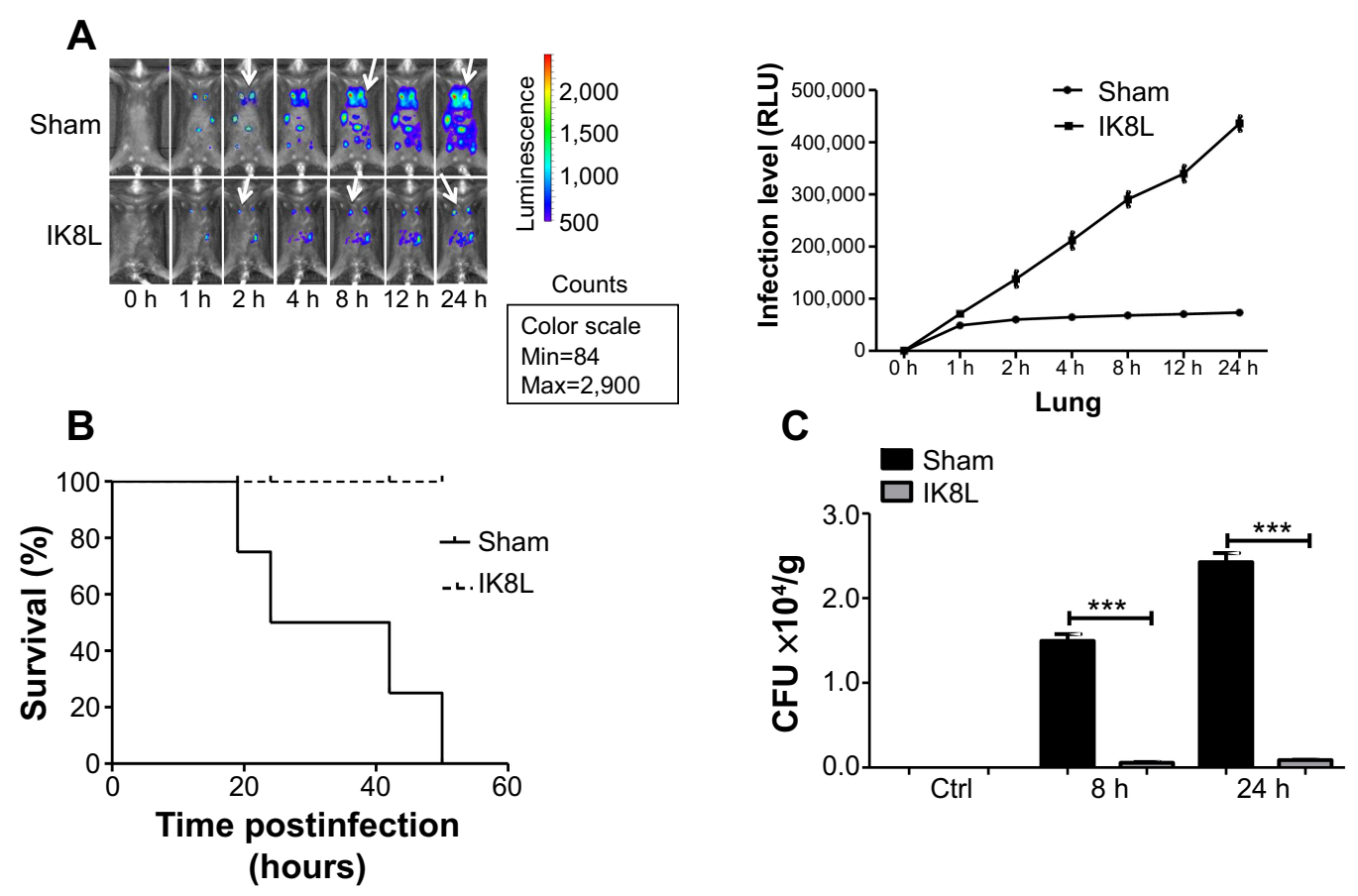

Figure I Peptide (IK8L) decreased mortality rates and disease severity in Kp-infected mice.

Notes: $(\mathbf{A})$ Whole animal imaging of bioluminescence was obtained using IVIS XRII system at different time points. Sham- and IK8L-treated mice were infected with I $\times$ I $^{5}$ CFU of Kp Xen-39 by nasal cavity (Caliper) (arrows indicating Kp spread regions). (B) Kaplan-Meier survival curves were obtained ( $P=0.0455$; $95 \%$ confidence interval: I.04I to 52.46 , log-rank test). (C) Bacterial burdens (CFU) of $K p$-infected mice treated with IK8L or control at different times. In $(\mathbf{A})$, data are shown as mean \pm SD/SEM of $n=4$ mice per group; $(\mathbf{A}-\mathbf{C})$ are representative of four mice per group. $* * * P<0.00 I$; Mann-Whitney $U$-test.

Abbreviations: CFU, colony-forming units; h, hours; Kp Xen-39, ; Max, maximum; Min, minimum; RLU, relative LUC units; LUC, luminescence unit counts; SD, standard deviation; SEM, standard error of the mean.

24 hours postinfection (Figure 1B), and all infected mice died at 50 hours. In contrast, all mice receiving IK8L treatment remained alive during the entire period of observation (Figure 1B). These results suggest that IK8L treatment is effective and improves the survival chances of infected mice. Furthermore, the lung homogenates were investigated for bacterial burdens. IK8L-treated mice exhibited significantly decreased CFUs of $K p$ compared to the control mice without IK8L treatment at both 8 hours and 24 hours postinfection (Figure 1C).

\section{IK8L reduced AM killing after $K p$ infection in mice}

Since $K p$ Xen-39 was an engineered bioluminescent bacterium for imaging analysis, we next chose the widely used $K p$ and $K p$-GFP strain to explore the pathogenesis mechanisms of IK8L against $K p$ infection. AM cells are key immune cells against Gram-negative bacterial infection. AM cells were isolated from the infected mice using BAL and evaluated for their viability. The viability of AM was increased by approximately threefold in IK8L-treated mice compared to untreated mice both at 24 hours and 48 hours postinfection (Figure 2A), indicating that IK8L treatment increased AM survival during $K p$ infection. PMN in the lung and blood was analyzed at 8 hours and 24 hours postinfection. ${ }^{18}$ Cell apoptosis was determined by flow cytometry using Annexin V/propidium iodide double staining, which showed a significant decrease in the apoptotic population in IK8L-treated group (3.23\%) compared to the sham control group $(51.53 \%$ ) (Figure 2B). PMN penetration was also lower in both the BAL fluid and blood of IK8L-treated mice than that of sham-treated mice (Figure $2 \mathrm{C}$ and D). In addition, PMN in the BAL fluid of the control mice increased with time, while that in the peptide-treated mice was effectively suppressed from 24 to 48 hours postinfection (Figure 2C). As phagocyte-derived reactive oxygen species (ROS) is of crucial importance against bacterial infections, ${ }^{39}$ ROS levels in AM cells of $K p$-infected mice were assessed at 8 hours and 24 hours postinfection by an NBT assay. ROS was decreased in AM cells from IK8L-treated mice compared to control mice, which might lead to reduced production of superoxide dismutase (Figure 2E). To further ascertain this finding, a sensitive assay, $\mathrm{H}_{2}$ DCF-DA, was also used to quantify superoxide in AMs. Indeed, the production of superoxide in IK8L-treated mice was reduced compared to sham-treated mice (Figure 2F). 

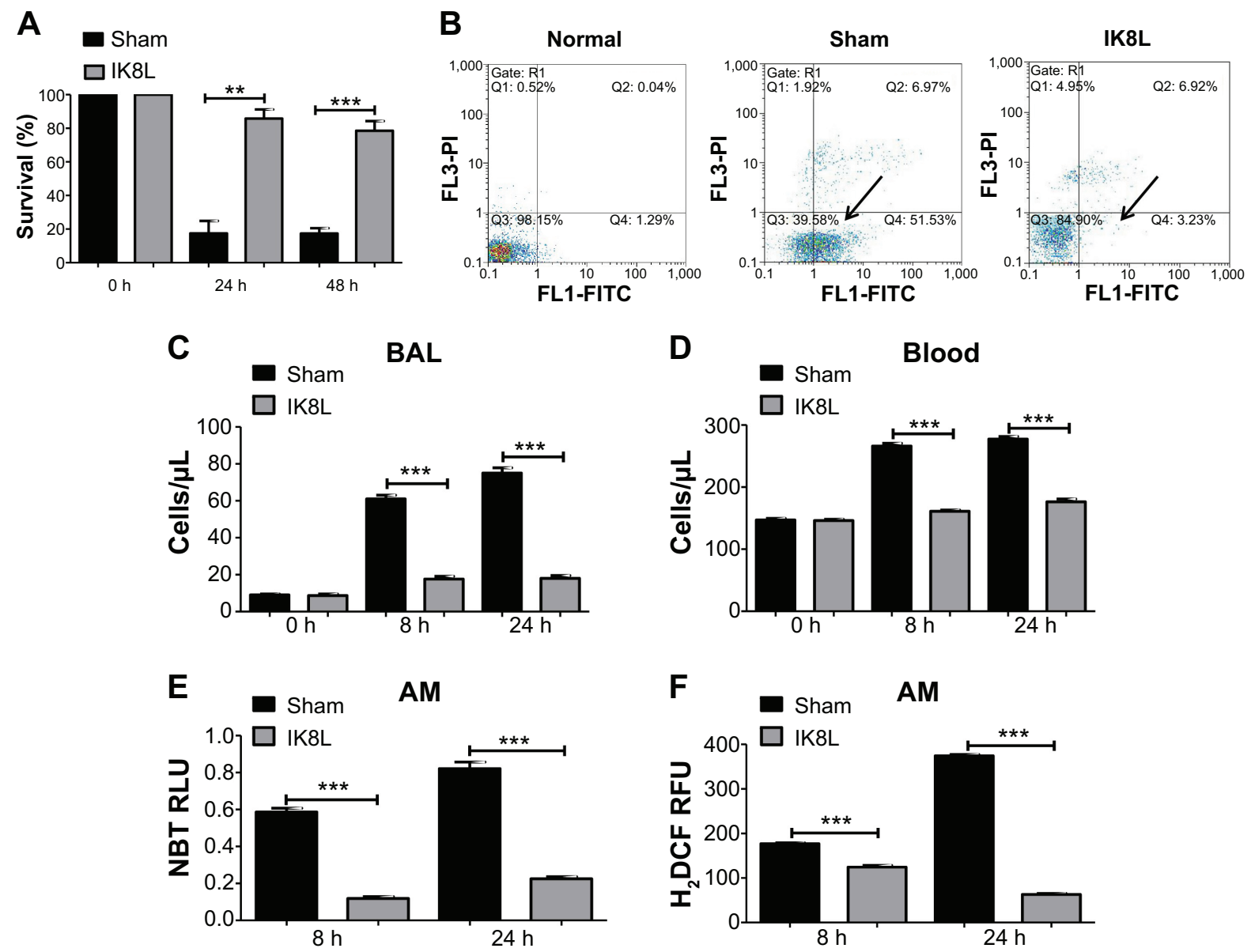

Figure $2 \mathrm{IK} 8 \mathrm{~L}$ increased AM viability in Kp-infected mice.

Notes: (A) Cell viability was determined in AMs by MTT assay. AM cells were obtained separately from infected mice and IK8L-treated mice. The absorbance of each sample was recorded at $560 \mathrm{~nm}$ to determine the cell viability rate. (B) Scattered distribution graph of apoptotic staining of the AM cells with or without IK8L treatment. The data of apoptotic percentage of treated cells vs the control are representative of two experiments (arrows indicating the apoptotic levels). (C and D) PMN infiltration in the BAL and blood was counted by Hema staining (Thermo Fisher, Waltham, MA, USA). (E) Superoxide production in AM cells detected using an NBT assay. (F) Oxidative stress in AM cells was determined by $\mathrm{H}_{2} \mathrm{DCF}$ assay. Data are shown as mean $\pm \mathrm{SEM}$ of $\mathrm{n}=3$ samples and are representative of three independent experiments. $* * P<0.0 \mathrm{I}$; $* * * P<0.00 \mathrm{I}$; one-way ANOVA (Tukey's post hoc).

Abbreviations: AM, alveolar macrophage; ANOVA, analysis of variance; BAL, bronchoalveolar lavage; h, hours; $\mathrm{H}_{2} \mathrm{DCF}$, dihydrodichlorofluorescein diacetate; Kp, Klebsiella pneumoniae; MTT, 3-(4,5-dimethyl-2-thiazolyl)-2,5 diphenyltetrazolium bromide; NBT, nitroblue tetrazolium; PMN, polymorphonuclear neutrophil; Q, quarter; RFU, relative fluorescence units; RLU, relative LUC units; SEM, standard error of the mean.

\section{IK8L decreased infection-induced tissue injury}

To further assess the tissue damage, the morphological alterations in the lung of infected mice were next investigated by H\&E staining. As shown in Figure 3A, the infected mice without sham-treated bacteria exhibited apparent signs of inflammatory response, tissue damage, and severe pneumonia. In contrast, the lungs of IK8L-treated mice had lower inflammatory features, including PMN penetration and tissue damage, than the sham-treated controls. Another mortiferous factor for bacterial infection is bacterial dissemination from lungs to other organs. Bacterial dissemination can promote disease progression, such as bacteremia and ultimately sepsis. ${ }^{39}$ Therefore, bacterial burdens in the liver, spleen, and kidney were further examined. As compared to the lungs (Figure 1C), bacterial load magnitudes in these organs were decreased (Figure S1A-C). Particularly, bacterial CFUs were significantly decreased in the liver, spleen, and kidney of IK8L-treated mice compared to those of sham-treated mice (Figure S1A-C). Myeloperoxidase (MPO) is released from PMN in the lung tissue in response to exposure to various pulmonary insults, ${ }^{40}$ which may serve as an indicator for infection extent. The MPO activity of the lung and other organs was analyzed, and it was found that MPO activity of the lung, liver, spleen, and kidney in IK8L-treated mice was significantly lower than that of sham-treated mice (Figure 3B-E). These findings suggest that IK8L treatment reduced organ damage induced by $K p$ infection. 

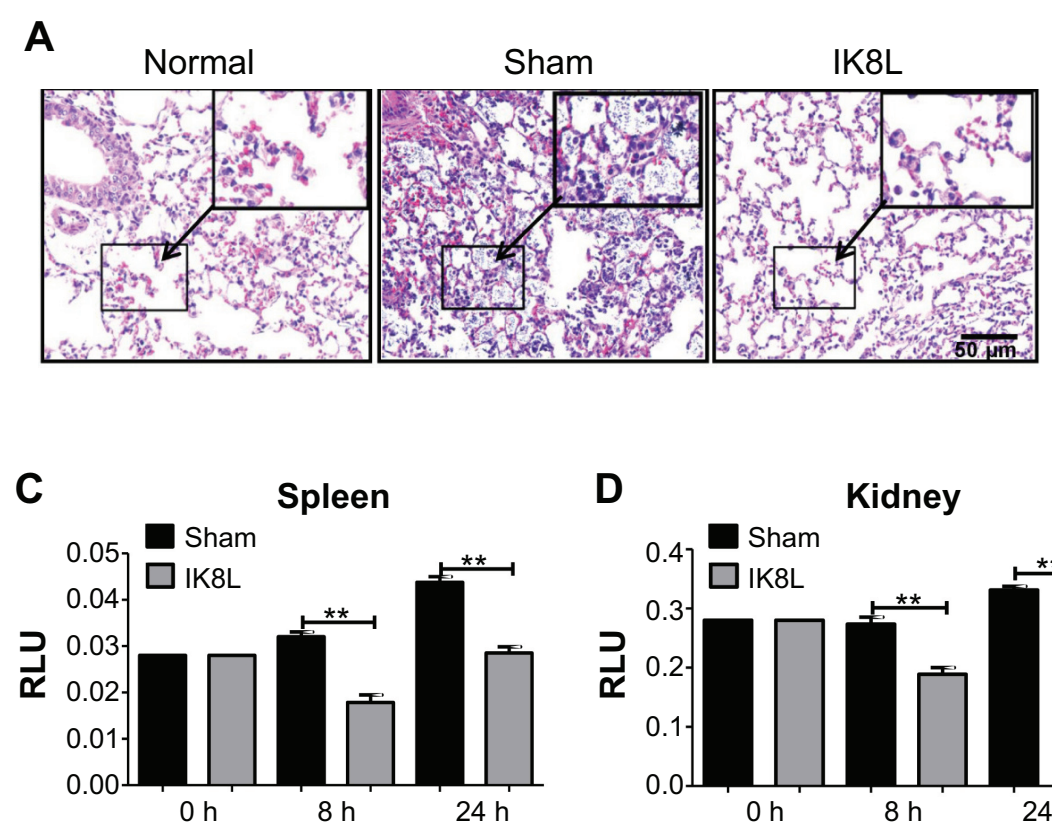

D

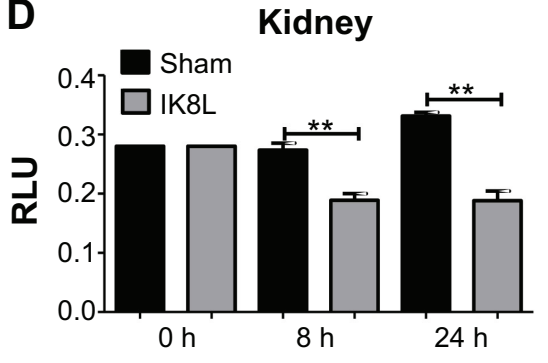

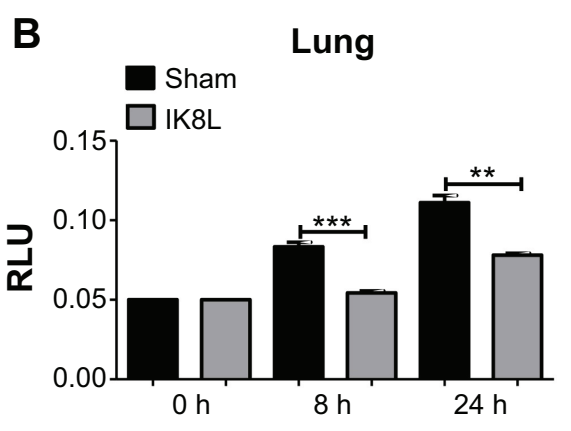

E

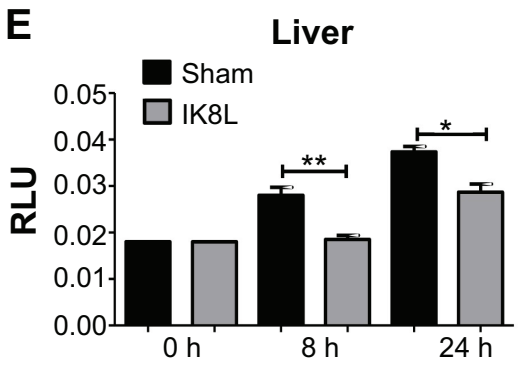

Figure $3 \mathrm{IK} 8 \mathrm{~L}$ decreased infection-induced tissue injury.

Notes: (A) Morphological evidence of lung injury and inflammatory responses as assessed by H\&E staining histological analysis (20X, scale bar $=50 \mu \mathrm{m}$, inset shows the typical tissue injury and inflammatory influx). (B-E) Decreased MPO in the lung, kidney, spleen, and liver of IK8L-treated mice. Data are shown as mean \pm SEM of $n=3$ mice per group and are representative of three independent experiments. $* P<0.05 ; * * P<0.01 ; * * * P<0.00$ I; one-way ANOVA (Tukey's post hoc).

Abbreviations: ANOVA, analysis of variance; h, hours; H\&E, hematoxylin and eosin; MPO, myeloperoxidase; RLU, relative LUC units; SEM, standard error of the mean.
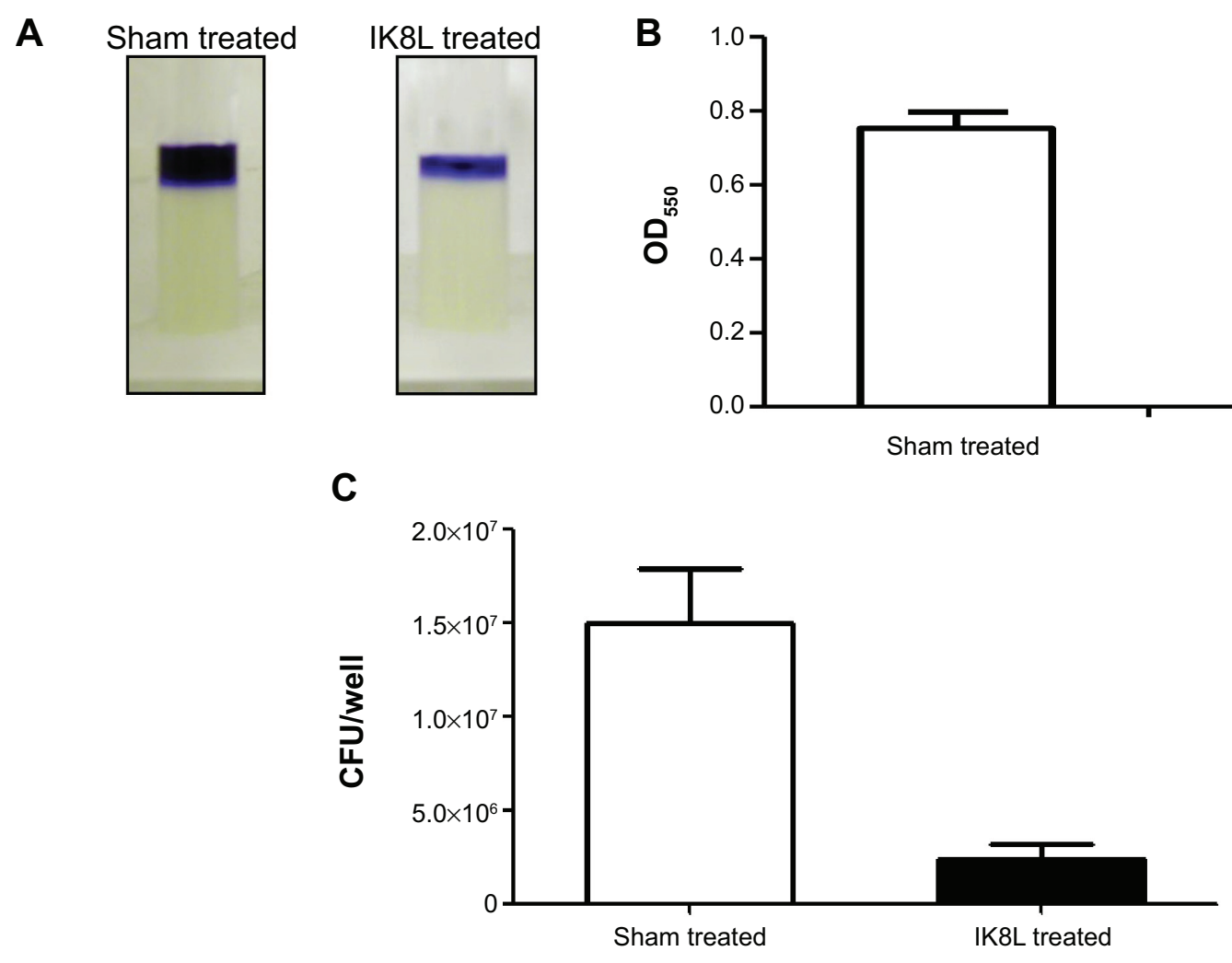

Figure $4 \mathrm{IK} 8 \mathrm{~L}$ killed $\mathrm{Kp}$ by blocking biofilm formation.

Notes: (A) Biofilm formation assessed by crystal violet staining of biofilms grown in microtiter wells for 8 hours. (B) Quantification of staining of wells in (A) after solubilization with acetic acid and measurement of $\mathrm{OD}_{550^{\circ}}$ (C) CFU per well of biofilm bacteria.

Abbreviations: $\mathrm{CFU}$, colony-forming units; $\mathrm{Kp}$, Klebsiella pneumoniae; $\mathrm{OD}_{550}$, optical density at $550 \mathrm{~nm}$. 
IK8L killed $K p$ by blocking biofilm formation and modulated $K p$-induced inflammatory responses via the IL- 6 signaling pathway

To explore the molecular mechanism in killing $K p$, we detected biofilms of $K p$ and observed its inability to form biofilms when treated with IK8L (Figure 4A-C, compared to sham controls). Microscopy observation indicated that the biofilm of $K p$ was thinner in IK8L-treated groups than sham controls. Thus, these data suggest that IK8L may interfere with the biological behavior of $K p$, hampering biofilm formation. We speculate that IK8L-mediated bactericidal activity is at least partially related to biofilm formation.

To analyze whether IK8L may regulate inflammation during $K p$ infection, we assessed various cytokines in BALF by
ELISA. ${ }^{41}$ The levels of tumor necrosis factor alpha (TNF- $\alpha$ ), interleukin-6 (IL-6), and IL-1 $\beta$ decreased significantly in the BALF of IK8L-treated mice compared to those of shamtreated mice at 24 hours postinfection (Figure $5 \mathrm{~A}-\mathrm{C}$ ). To further explore the molecular bactericidal mechanism of IK8L in $\mathrm{Kp}$-infected mice, inflammation-relevant signaling proteins in lung homogenates were next analyzed. First, the expression of STAT3, JAK2, and ERK1/2 involved in the IL-6 signaling pathway was examined. $K p$ infection increased the expression of STAT3, JAK2, and ERK1/2 in the mice compared to sham-treated mice. However, IK8L treatment significantly reduced the levels of STAT3, JAK2, and ERK1/2 compared to sham-treated mice, and STAT3 was even lower than in untreated mice (Figure 5D). The expression and phosphorylation of $\mathrm{JNK}, \mathrm{NF}-\kappa \mathrm{B}$, and $\mathrm{I} \kappa \mathrm{B}$ was also measured, which
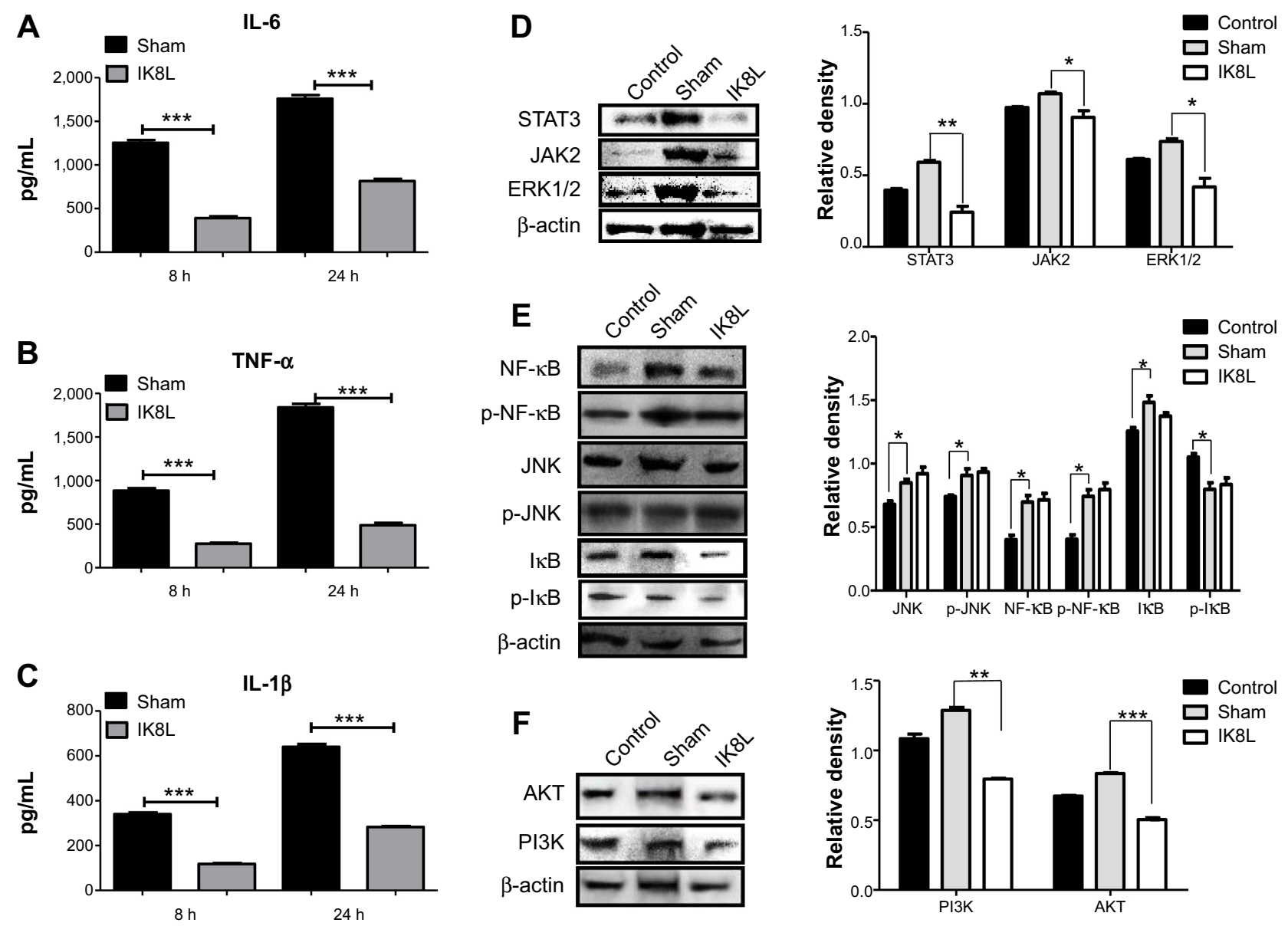

Figure $5 \mathrm{IK} 8 \mathrm{~L}$ modulated $\mathrm{Kp}$-induced inflammatory reaction via the IL-6/JAK/STAT3 signaling pathway.

Notes: (A-C) ELISA was used to measure IL-6, TNF- $\alpha$ and IL-I $\beta$ in BAL fluids of mice 8 hours and 24 hours post infection, respectively. (D) Cell signaling proteins relating to the JAK2/STAT3 signaling pathway were evaluated in lung tissue by Western blotting analysis. (E) Expression and phosphorylation of signaling proteins relating to NF- $\mathrm{B}$ transcription factor were evaluated in lung tissue by Western blotting. (F) Cell signaling proteins relating to the AKT signaling pathway were evaluated by Western blot. $\beta$-actin was used as a loading control. Data are shown as mean $\pm S E M$ of $n=3$ mice per group and are representative of three independent experiments. ${ }^{*} P<0.05$; ${ }^{*} * P<0.0$ I; $* * * P<0.00$ I; one-way ANOVA (Tukey's post hoc).

Abbreviations: ANOVA, analysis of variance; BAL, bronchoalveolar lavage; ELISA, enzyme-linked immunosorbent assay; IL, interleukin; Kp, Klebsiella pneumoniae; SEM, standard error of the mean; TNF- $\alpha$, tumor necrosis factor alpha; $p$, phosphorylation. 
showed that while the levels of JNK and IאB in IK8L-treated mice were similar to those of sham-treated mice following $K p$ infection, both protein expression and phosphorylation of $\mathrm{NF}-\kappa \mathrm{B}$ were increased by $K p$ infection and the increase was somewhat blocked by IK8L treatment (Figure 5E). These findings suggest that IK8L inhibited inflammatory responses by decreasing the expression of STAT3, JAK2, and ERK1/2, but did not inhibit NF- $\mathrm{B}$ transcription. To validate these observations, the expression of other signaling proteins (AKT and PI3K) was also measured, and also showed similar patterns as STAT3, JAK2, and ERK1/2 after IK8L treatment (Figure 5F). Taken together, IK8L modulated inflammatory responses during $K p$ infection through the JAK/STAT3 and AKT/PI3K signaling pathway.

\section{STAT3 signaling plays crucial roles in antibacterial activity of IK8L}

To further confirm the crucial role of JAK/STAT3 signaling, the STAT3 inhibitor was used to study the signal pathway in murine $\mathrm{MH}-\mathrm{S}$, a widely used model for studying murine macrophages function. ${ }^{42}$ After 3 hours incubation with this inhibitor, cells were infected with $K p$ for 1 hour at 10:1 MOI. As shown in Figure 6A, IK8L-treated cells exhibited markedly decreased production of ROS compared to sham-treated cells. However, blocking STAT3 increased production of ROS in IK8L-treated MH-S cells compared to control cells or IK8L-treated MH-S cells without blocking, as determined by an NBT assay (Figure 6A). Moreover, $\mathrm{H}_{2}$ DCF-DA assay was also used to measure superoxide for validating the NBT
A

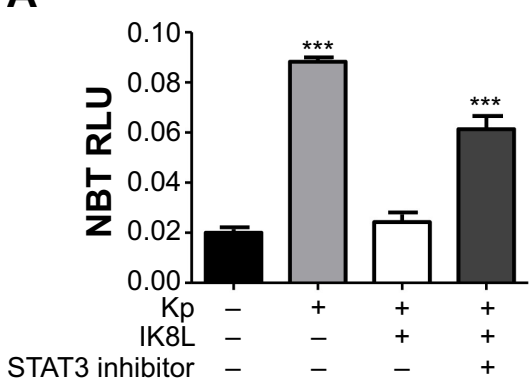

B

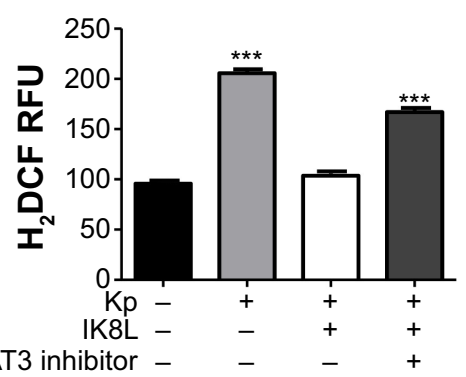

C
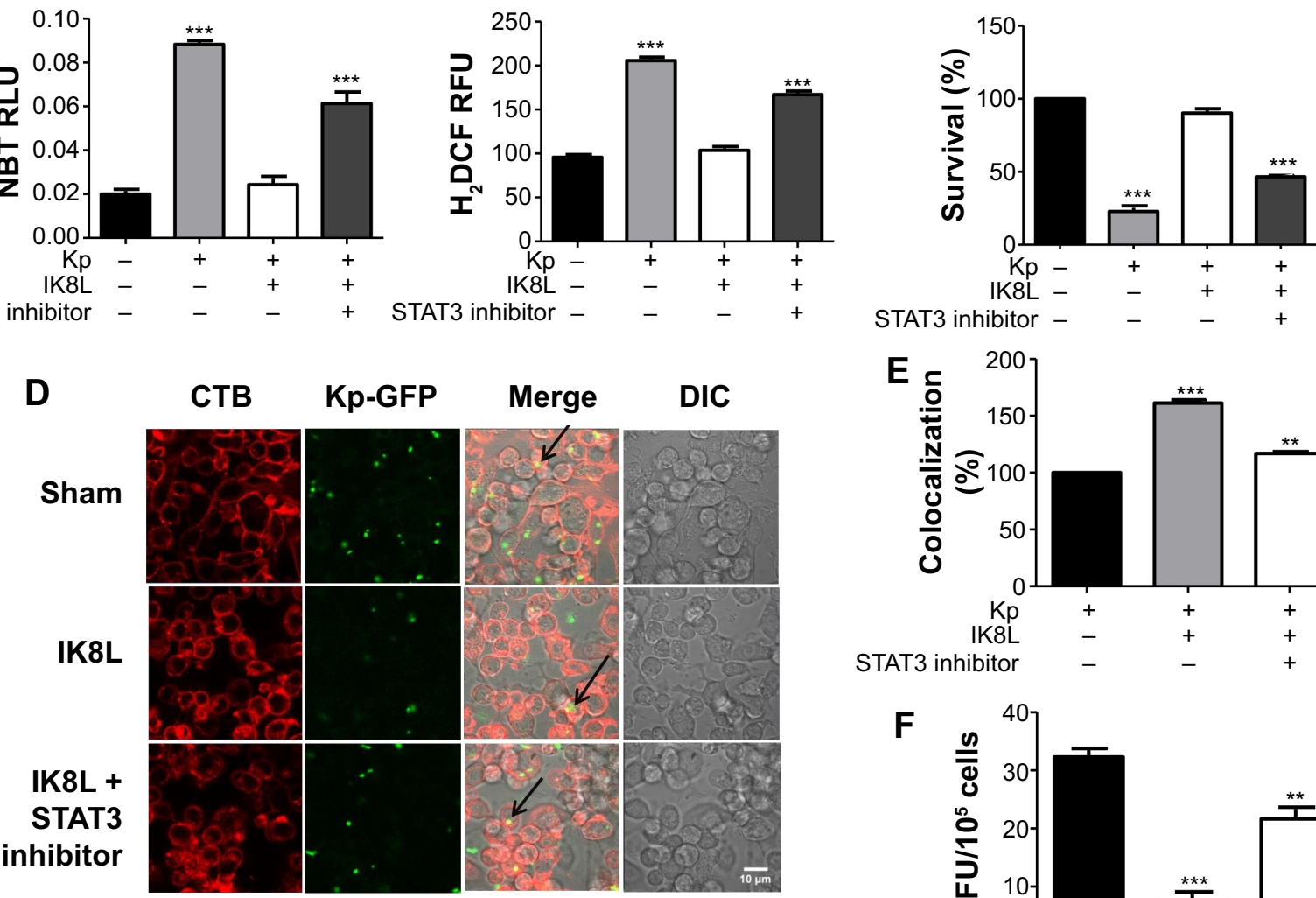
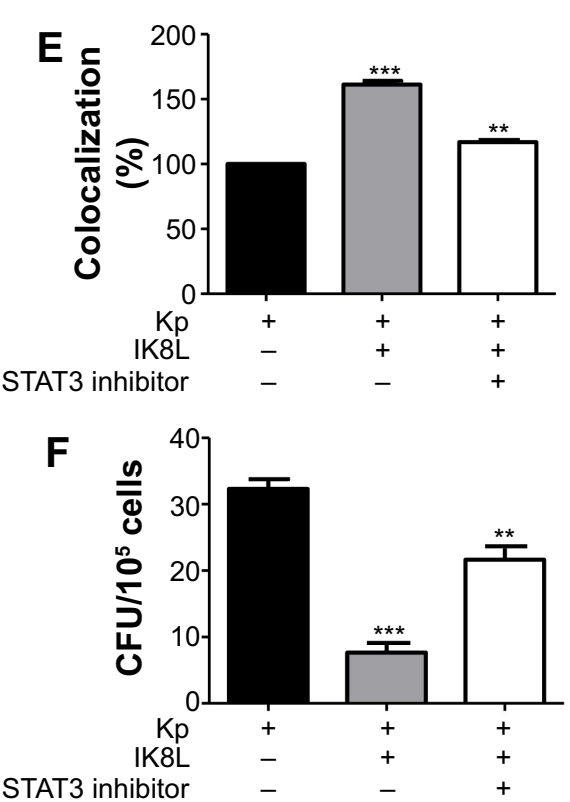

Figure 6 STAT3 signaling pathway played a crucial role in IK8L antibacterial function

Notes: (A) Superoxide production in MH-S cells significantly increased by blocking STAT3 with an inhibitor, compared to controls before peptide treatment using an NBT assay $(\mathrm{I} \mathrm{mg} / \mathrm{mL}$ ). The data were recorded at $560 \mathrm{~nm}$ absorbance. (B) Oxidative stress was increased by blocking STAT3 compared to controls before peptide treatment as determined by the $\mathrm{H}_{2} \mathrm{DCF}$ assay $(5 \mu \mathrm{M})$. The fluorescence was quantified at $488 \mathrm{~nm}$. (C) MTT assay indicated decreased cell viability of blocking STAT3 in MH-S cells compared to controls before peptide treatment. (D) Confocal fluorescence microscopy image showing Kp distributions and lipid raft staining using CTB chain (arrows indicating internalized $K p$, scale bar $=20 \mu \mathrm{m}$ ). (E) Quantification of colocalization between $K p$ and lipid raft from $D$, and expressed relative to sham-treated cell. (F) Bacterial burdens (CFU) of $K p$-infected cells treated with IK8L and STAT3 inhibitor. The data are mean \pm SEM and are representative of three independent experiments (one-way ANOVA [Tukey's post hoc]; asterisk compared with control; ** $P<0.01$; ***P<0.00I).

Abbreviations: ANOVA, analysis of variance; CFU, colony-forming units; CTB, cholera toxin B; RLU, relative LUC units; $\mathrm{H}_{2} D C F$, dihydrodichlorofluorescein diacetate; Kp, Klebsiella pneumoniae; MH-S, murine alveolar macrophage cell line; MTT, 3-(4,5-dimethyl-2-thiazolyl)-2,5-diphenyltetrazolium bromide; NBT, nitroblue tetrazolium; RFU, relative fluorescence units; SEM, standard error of the mean; DIC, differential interference contrast. 
data, and IK8L treatment indeed reduced the production of superoxide, while blocking STAT3 led to increased superoxide production (Figure 6B). Furthermore, the viability of $K p$-infected cells was increased after IK8L treatment, while blocking STAT3 reduced cell viability compared to IK8L-treated cells as assessed by an MTT assay (Figure $6 \mathrm{C})$. In vivo dynamic analysis also indicates the potential role of IK8L in eradicating $K p$ in $\mathrm{MH}-\mathrm{S}$ cells. MH-S cells were treated with IK8L $(62.5 \mu \mathrm{g} / \mathrm{mL})$ for 4 hours; after 3 hours of blocking, cells were infected with $K p$-GFP in a 10:1 MOI for 2 hours. Then the cells were stained with lipid raft marker rhodamine-labeled CTB. Lipid rafts are shown to be associated with membrane signaling, including phagocytosis. ${ }^{43,44}$ The distribution of $K p$ within these cells was evaluated using confocal laser-scanning microscopy, and the bacterial amount in IK8L-treated cells was less than that in cells without IK8L treatment. There are more lipid rafts aggregates (CTB stained) in IK8L-treated cells than sham-treated cells, indicating that more active lipid rafts may contribute to enhanced antibacterial potency (Figure $6 \mathrm{D})$. Thus, we quantified the colocalization between invading $K p$ and lipid rafts, which showed significant overlaps in IK8L-treated cells than sham-treated cells (Figure 6E). These data suggest that increased raft aggregates may facilitate bacterial killing by IK8L. Importantly, blocking STAT3 resulted in bacterial amounts similar to that in sham-treated groups (Figure 5D). To more quantitatively analyze the data, cell lysates were used to enumerate bacterial burdens, which were in good agreement with the results of microscopy analysis (Figure 6F). These results indicated that IK8L markedly inhibited $K p$ survival rates in mouse macrophage cells. However, blocking STAT3 also increased $K p$ survival rates compared to controls without using STAT3 inhibitor, implying that IK8L inhibited $K p$ survival rates in a JAK/STAT3-dependent manner. Taken together, JAK/STAT3 signaling probably plays an essential role in the antimicrobial function against $K p$, which is delineated in a schematic diagram (Figure 7).

\section{Discussion}

Recently we have designed a series of short synthetic $\beta$-sheet folding peptides with $\left(\mathrm{X}_{1} \mathrm{Y}_{1} \mathrm{X}_{2} \mathrm{Y}_{2}\right)$ n sequence $(\mathrm{X}$ : hydrophobic amino acids, Y: cationic amino acid, $n$ : the number of repeat units). By disrupting the bacterial membrane, IK8L

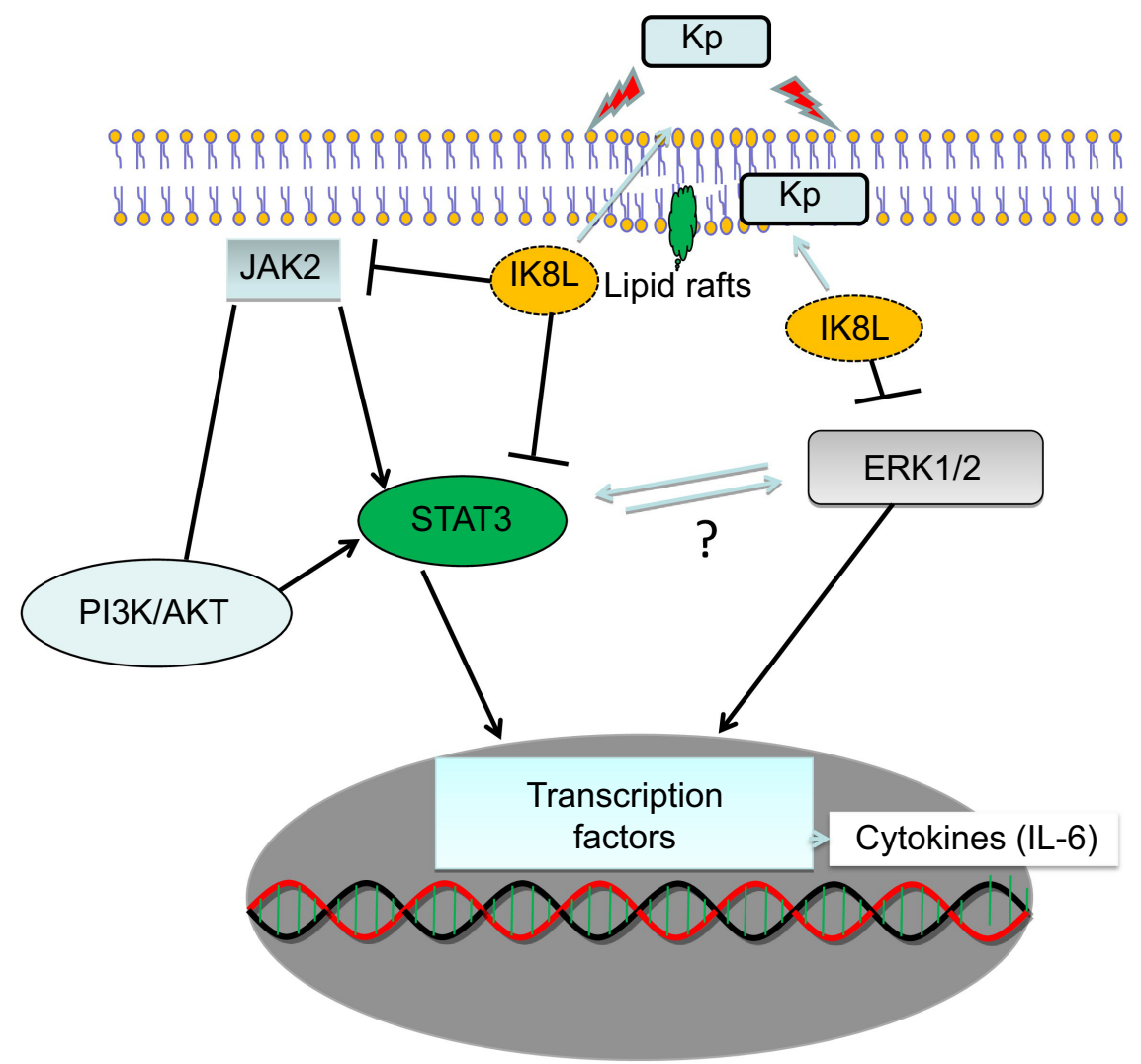

Figure 7 Schematic diagram showing how IK8L regulates the JAK/STAT3 pathway and counteracts the Klebsiella infection, especially through modulation of inflammatory responses.

Abbreviations: IL, interleukin; Kp, Klebsiella pneumoniae. 
effectively killed clinically isolated multidrug-resistant bacteria, such as methicillin-resistant Staphylococcus aureus, Acinetobacter baumannii, Pseudomonas aeruginosa, and Mycobacterium tuberculosis, ${ }^{45}$ and prevented drug resistance development. Significantly reduced intracellular bacteria counts were observed after IK8L treatment in murine macrophage cell line RAW264.7 with Staphylococcus aureus infection. IK8L was found to be optimal with abilities of endotoxin neutralization and biofilm eradication. ${ }^{21}$ To investigate whether this peptide has potential for use as an antibacterial agent against the difficult-to-kill $K p$ infection in clinics, the efficacy of IK8L against $K p$-induced acute pneumonia and its role in the host defense were studied using our well-established mouse model. ${ }^{46}$ Our data demonstrated that IK8L significantly alleviated severe infection after lethal dose challenge in mice, leading to significantly reduced bacterial burdens in major organs, decreased mortality, and lowered inflammatory cytokine response. Furthermore, these studies suggest that IK8L may be able to eradicate the invading bacteria, thereby attenuating inflammatory responses and avoiding tissue injury.

Infection of MDR bacteria has become a global health issue in both communities and hospitals. ${ }^{47}$ The evolution of clinical strains of antibiotic-resistant bacteria has significantly increased the risk of hospital-acquired infection. ${ }^{48}$ The ineffectiveness of commonly used antibacterial drugs and the emergence of antibiotic-resistant bacteria require new potent therapy for drug-resistant infections. One innovative approach is to use AMPs with a membrane-lytic functional mechanism as antibiotic agents.

In this study, the bactericidal activity of IK8L was evaluated in mouse models. This peptide targeted bacterial outer membrane structure and showed surprisingly high efficacy in killing MDR Gram-positive and Gram-negative bacteria. ${ }^{38}$ Compared to other antibacterial peptides, IK8L shows high sterilization activity with a broad spectrum. One typical antibacterial mechanism of IK8L is the membrane disruption. The membrane of $K p$, a successful Gram-negative human pathogen, contains abundantly charged lipids, and these negative charges can significantly enhance the membrane binding of peptide. ${ }^{49}$ Sepsis is a grave clinical syndrome due to high mortality rates, resulting from an impaired host response to infection. ${ }^{50}$ An obvious increase in the survival rate of $\mathrm{Kp}$-infected mice has, to our knowledge, been observed for the first time, indicating high efficacy of IK8L against $K p$ infection in a time- and dose-dependent manner. In a mouse septic model, groups treated with IK8L significantly reduced infection levels. In the present study, a dose of $20 \mathrm{mg} / \mathrm{kg}$ was highly effective in vivo when administered intravenously. Meanwhile, we also used a math model to gauge infection levels over long time periods, which might be a potential tool to predict disease progression of $K p$ infection in clinics.

We also found that IK8L could slow down the formation of biofilms. As biofilm is very important for bacterial resistance to antibiotics and a harsh host environment, the peptide may be useful for controlling chronic infection in immunodeficient individuals. Another key finding of our study is that IK8L could markedly decrease cytokine response IL-6 in mice infected by $K p$, which was observed both in the lungs and BAL fluids. In this model, IL-6 was down-regulated through a STAT3 pathway in IK8L-treated mice. Other reports have shown that IL- $1 \beta$ and TNF- $\alpha$ were important for host responses to $K p$ infection. ${ }^{51}$ However, we have found the changes of these two cytokines were less substantive. The detailed mechanism is unknown but might be due to a select impact on the production of proinflammatory cytokines in $K p$-infected mice. $K p$ infection can significantly promote a spontaneous activation of the JAK/STAT pathway. However, the IK8L can downregulate STAT3 and ERK1/2 expression in $K p$ infected cells and organs, and subsequently inhibit the downstream signaling pathway factors and inflammatory reaction. These findings suggest that IK8L did not inhibit NF- $\kappa \mathrm{B}$ transcription and $\mathrm{AKT} / \mathrm{PI} 3 \mathrm{~K}$ expression following infection. Thus, we speculate that there may be an interaction between STAT3 and ERK1/2 to impact proinflammatory cytokine production. However, how these signaling pathways regulate inflammatory responses is still unclear and needs additional validations, which may be interesting future directions for further research.

Due to the complexity of cell signals that may constitute a variety of cellular components and organ systems, ${ }^{52,53}$ much needs to be pursued in the future to further understand the detailed mechanisms after in vivo peptide application. We used the peritoneal route to administer the peptide, but airway delivery may also be considered for preventing or treating lung infection, a frequent invasion route of $K p$. It will also be interesting to test the applicability of the peptide in treatment of $P$. aeruginosa in animals, another successful bacterium crying for lack of effective vaccines. ${ }^{54,55}$ Although current studies indicate a potential for the peptide to be applied in prevention and treatment of $K p$ infection, we predict that there may be a long process before the realization of that promise, like other preventive and therapeutic research. ${ }^{53,56-59}$ 


\section{Conclusion}

The current study has demonstrated that IK8L is highly effective against $K p$ infection in an infected- $K p$ mouse model. Since the ever-increasing multidrug resistance limits available therapeutic options, this novel AMP may provide a strategy for treating $K p$ or other MDR infections. Future studies should attempt to fully characterize the usefulness of antimicrobial IK8L as a clinically useful therapeutic for multidrug-resistant infectious diseases.

\section{Acknowledgments}

This work was funded by the Flight Attendant Medical Research Institute (FAMRI, Grant \#103007), National Institute of Health AI109317-01A1, AI101973-01, AI09753201A1, National Natural Science Foundation of China (31260276, 30760057, and 30960091), Yunnan Province Science and Technology Innovation Team (2011CI123, 2012Z053, and 2012S208), and Institute of Bioengineering and Nanotechnology (Biomedical Research Council, Agency for Science, Technology and Research, Singapore).

\section{Author contributions}

Shirui Tan and Changpei Gan contributed equally to this work; thus, both are co-first authors. All authors contributed toward data analysis, drafting, and revising the paper and agree to be accountable for all aspects of the work.

\section{Disclosure}

The authors declare no conflicts of interest in this work.

\section{References}

1. Lee JK, Park SC, Hahm KS, Park Y. A helix-PXXP-helix peptide with antibacterial activity without cytotoxicity against MDRPA-infected mice. Biomaterials. 2014;35(3):1025-1039.

2. Peters BM, Shirtliff ME, Jabra-Rizk MA. Antimicrobial peptides: primeval molecules or future drugs? PLoS Pathog. 2010;6(10):e1001067.

3. Pompilio A, Crocetta V, Scocchi M, et al. Potential novel therapeutic strategies in cystic fibrosis: antimicrobial and anti-biofilm activity of natural and designed $\alpha$-helical peptides against Staphylococcus aureus, Pseudomonas aeruginosa, and Stenotrophomonas maltophilia. BMC Microbiol. 2012;12:145.

4. Adachi R, Suzuki K. Lyn, one of the Src-family tyrosine kinases expressed in phagocytes, plays an important role in beta2 integrinsignalling pathways in opsonized zymosan-activated macrophage-like U937 cells. Cell Biochem Funct. 2007;25(3):323-333.

5. Lopes SP, Ceri H, Azevedo NF, Pereira MO. Antibiotic resistance of mixed biofilms in cystic fibrosis: impact of emerging microorganisms on treatment of infection. Int J Antimicrob Agents. 2012;40(3):260-263.

6. Lech M, Römmele C, Kulkarni OP, et al. Lack of the long pentraxin PTX3 promotes autoimmune lung disease but not glomerulonephritis in murine systemic lupus erythematosus. PLoS One. 2011;6(5):e20118.

7. van Zoelen MA, Verstege MI, Draing C, et al. Endogenous MCP-1 promotes lung inflammation induced by LPS and LTA. Mol Immunol. 2011;48(12-13):1468-1476.
8. Izbicka E, Streeper RT, Michalek JE, Louden CL, Diaz A, Campos DR. Plasma biomarkers distinguish non-small cell lung cancer from asthma and differ in men and women. Cancer Genomics Proteomics. 2012;9(1):27-35.

9. Thornton MM, Chung-Esaki HM, Irvin CB, Bortz DM, Solomon MJ, Younger JG. Multicellularity and antibiotic resistance in Klebsiella pneumoniae grown under bloodstream-mimicking fluid dynamic conditions. J Infect Dis. 2012;206(4):588-595.

10. Orsi GB, García-Fernández A, Giordano A, et al. Risk factors and clinical significance of ertapenem-resistant Klebsiella pneumoniae in hospitalised patients. $J$ Hosp Infect. 2011;78(1):54-58.

11. O'Neill AJ. New antibacterial agents for treating infections caused by multi-drug resistant Gram-negative bacteria. Expert Opin Investig Drugs. 2008;17(3):297-302.

12. Jenssen H, Hamill P, Hancock RE. Peptide antimicrobial agents. Clin Microbiol Rev. 2006;19(3):491-511.

13. Wiesner J, Vilcinskas A. Antimicrobial peptides: the ancient arm of the human immune system. Virulence. 2010;1(5):440-464.

14. Cederlund A, Gudmundsson GH, Agerberth B. Antimicrobial peptides important in innate immunity. FEBS J. 2011;278(20):3942-3951.

15. Harman GE, Howell CR, Viterbo A, Chet I, Lorito M. Trichoderma species - opportunistic, avirulent plant symbionts. Nat Rev Microbiol. 2004;2(1):43-56.

16. Rajanbabu V, Chen JY. Applications of antimicrobial peptides from fish and perspectives for the future. Peptides. 2011;32(2):415-420.

17. Cudic M, Bulet P, Hoffmann R, Craik DJ, Otvos L. Chemical synthesis, antibacterial activity and conformation of diptericin, an 82-mer peptide originally isolated from insects. Eur J Biochem. 1999; 266(2):549-558.

18. Yuan K, Huang C, Fox J, et al. Elevated inflammatory response in caveolin-1-deficient mice with Pseudomonas aeruginosa infection is mediated by STAT3 protein and nuclear factor kappaB (NF-kappaB). J Biol Chem. 2011;286(24):21814-21825.

19. Li X, Zhou X, Ye Y, et al. Lyn regulates inflammatory responses in

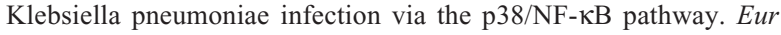
J Immunol. 2014;44(3):763-773.

20. Guo Q, Shen N, Yuan K, et al. Caveolin-1 plays a critical role in host immunity against Klebsiella pneumoniae by regulating STAT5 and Akt activity. Eur J Immunol. 2012;42(6):1500-1511.

21. Ong ZY, Cheng J, Huang Y, et al. Effect of stereochemistry, chain length and sequence pattern on antimicrobial properties of short synthetic $\beta$-sheet forming peptide amphiphiles. Biomaterials. 2014;35(4): $1315-1325$.

22. Zhou X, Li X, Ye Y, et al. MicroRNA-302b augments host defense to bacteria by regulating inflammatory responses via feedback to TLR/ IRAK4 circuits. Nat Commun. 2014;5:3619.

23. O'Toole GA, Pratt LA, Watnick PI, Newman DK, Weaver VB, Kolter R. Genetic approaches to study of biofilms. Methods Enzymol. 1999; 310:91-109.

24. Deng X, Weerapana E, Ulanovskaya O, et al. Proteome-wide quantification and characterization of oxidation-sensitive cysteines in pathogenic bacteria. Cell Host Microbe. 2013;13(3):358-370.

25. Wu M, Kelley MR, Hansen WK, Martin WJ. Reduction of BCNU toxicity to lung cells by high-level expression of $\mathrm{O}(6)$-methylguanine-DNA methyltransferase. Am J Physiol Lung Cell Mol Physiol. 2001;280(4): L755-L761.

26. Kannan S, Huang H, Seeger D, et al. Alveolar epithelial type II cells activate alveolar macrophages and mitigate $P$. aeruginosa infection. PLoS One. 2009;4(3):e4891.

27. Wu M, Audet A, Cusic J, Seeger D, Cochran R, Ghribi O. Broad DNA repair responses in neural injury are associated with activation of the IL-6 pathway in cholesterol-fed rabbits. J Neurochem. 2009;111(4): 1011-1021.

28. Wu M, Huang $\mathrm{H}$, Zhang $\mathrm{W}$, et al. Host DNA repair proteins in response to Pseudomonas aeruginosa in lung epithelial cells and in mice. Infect Immun. 2011;79(1):75-87. 
29. Long Q, Huang W, Yao Y, et al. Virus-like particles presenting interleukin-33 molecules: Immunization characteristics and potentials of blocking IL-33/ST2 pathway in allergic airway inflammation. Hum Vaccin Immunother. 2014;10(8):2303-2311.

30. Gast A, Anderson W, Probst A, et al. Pharmacokinetics and distribution of recombinant secretory leukocyte proteinase inhibitor in rats. Am Rev Respir Dis. 1990;141(4 Pt 1):889-894.

31. Wu M, Stockley PG, Martin WJ. An improved western blotting technique effectively reduces background. Electrophoresis. 2002;23(15): 2373-2376.

32. Yuan K, Huang C, Fox J, et al. Autophagy plays an essential role in the clearance of Pseudomonas aeruginosa by alveolar macrophages. $J$ Cell Sci. 2012;125(Pt 2):507-515.

33. Li G, Yuan K, Yan C, et al. 8-Oxoguanine-DNA glycosylase 1 deficiency modifies allergic airway inflammation by regulating STAT6 and IL-4 in cells and in mice. Free Radic Biol Med. 2012;52(2):392-401.

34. Ye Y, Wang H, Chu JH, et al. Atractylenolide II induces G1 cell-cycle arrest and apoptosis in B16 melanoma cells. J Ethnopharmacol. 2011; 136(1):279-282.

35. Kannan S, Pang H, Foster DC, Rao Z, Wu M. Human 8-oxoguanine DNA glycosylase increases resistance to hyperoxic cytotoxicity in lung epithelial cells and involvement with altered MAPK activity. Cell Death Differ. 2006;13(2):311-323.

36. He YH, Wu M, Kobune M, Xu Y, Kelley MR, Martin WJ. Expression of yeast apurinic/apyrimidinic endonuclease (APN1) protects lung epithelial cells from bleomycin toxicity. Am J Respir Cell Mol Biol. 2001;25(6):692-698.

37. Lu Z, Dai T, Huang L, et al. Photodynamic therapy with a cationic functionalized fullerene rescues mice from fatal wound infections. Nanomedicine (Lond). 2010;5(10):1525-1533.

38. Ong ZY, Gao SJ, Yang YY. Short synthetic $\beta$-sheet forming peptide amphiphiles as broad spectrum antimicrobials with antibiofilm and endotoxin neutralizing capabilities. Adv Funct Mater. 2013;23(29): 3682-3692.

39. Reino DC, Palange D, Feketeova E, et al. Activation of toll-like receptor 4 is necessary for trauma hemorrhagic shock-induced gut injury and polymorphonuclear neutrophil priming. Shock. 2012;38(1): 107-114.

40. Gonzalez RJ, Weening EH, Frothingham R, Sempowski GD, Miller VL. Bioluminescence imaging to track bacterial dissemination of Yersinia pestis using different routes of infection in mice. BMC Microbiol. 2012; $12: 147$.

41. Feyler A, Voho A, Bouchardy C, et al. Point: myeloperoxidase463G $->$ a polymorphism and lung cancer risk. Cancer Epidemiol Biomarkers Prev. 2002;11(12):1550-1554.

42. Regueiro V, Moranta D, Frank CG, et al. Klebsiella pneumoniae subverts the activation of inflammatory responses in a NOD1-dependent manner. Cell Microbiol. 2011;13(1):135-153.
43. Kannan S, Audet A, Huang H, Chen LJ, Wu M. Cholesterol-rich membrane rafts and Lyn are involved in phagocytosis during Pseudomonas aeruginosa infection. J Immunol. 2008;180(4):2396-2408.

44. Simons K, Ikonen E. How cells handle cholesterol. Science. 2000; 290(5497):1721-1726.

45. Davies J. Origins and evolution of antibiotic resistance. Microbiologia. 1996;12(1):9-16.

46. Chiavolini D, Pozzi G, Ricci S. Animal models of Streptococcus pneumoniae disease. Clin Microbiol Rev. 2008;21(4):666-685.

47. Mbawuike IN, Herscowitz HB. MH-S, a murine alveolar macrophage cell line: morphological, cytochemical, and functional characteristics. J Leukoc Biol. 1989;46(2):119-127.

48. Coque TM, Baquero F, Canton R. Increasing prevalence of ESBL-producing Enterobacteriaceae in Europe. Euro Surveill. 2008;13(47):1-11.

49. Wu G, Li X, Deng X, et al. Protective effects of antimicrobial peptide $\mathrm{S}$-thanatin against endotoxic shock in mice introduced by LPS. Peptides. 2011;32(2):353-357.

50. Wu G, Wu P, Xue X, et al. Application of S-thanatin, an antimicrobial peptide derived from thanatin, in mouse model of Klebsiella pneumoniae infection. Peptides. 2013;45:73-77.

51. Ye P, Garvey PB, Zhang P, et al. Interleukin-17 and lung host defense against Klebsiella pneumoniae infection. Am J Respir Cell Mol Biol. 2001;25(3):335-340.

52. Kannan S, Wu M. Respiratory stem cells and progenitors: overview, derivation, differentiation, carcinogenesis, regeneration and therapeutic application. Curr Stem Cell Res Ther. 2006;1(1):37-46.

53. Wu M. DNA repair proteins as molecular therapeutics for oxidative and alkylating lung injury. Curr Gene Ther. 2005;5(2):225-236.

54. Wang P, Wang X, Yang X, Liu Z, Wu M, Li G. Budesonide suppresses pulmonary antibacterial host defense by down-regulating cathelicidinrelated antimicrobial peptide in allergic inflammation mice and in lung epithelial cells. BMC Immunol. 2013;14:7.

55. Priebe GP, Goldberg JB. Vaccines for Pseudomonas aeruginosa: a long and winding road. Expert Rev Vaccines. 2014;13(4):507-519.

56. Yan J, Liu X, Wang Y, et al. Enhancing the potency of HBV DNA vaccines using fusion genes of HBV-specific antigens and the N-terminal fragment of gp96. J Gene Med. 2007;9(2):107-121.

57. Zhao K, Deng X, He C, Yue B, Wu M. Pseudomonas aeruginosa outer membrane vesicles modulate host immune responses by targeting the Toll-like receptor 4 signaling pathway. Infect Immun. 2013;81(12): 4509-4518.

58. Wu M, Pasula R, Smith PA, Martin WJ. Mapping alveolar binding sites in vivo using phage peptide libraries. Gene Ther. 2003;10(17) 1429-1436.

59. Zhang D, Wu M, Nelson DE, Pasula R, Martin WJ. Alpha-1-antitrypsin expression in the lung is increased by airway delivery of gene-transfected macrophages. Gene Ther. 2003;10(26):2148-2152. 


\section{Supplementary materials}
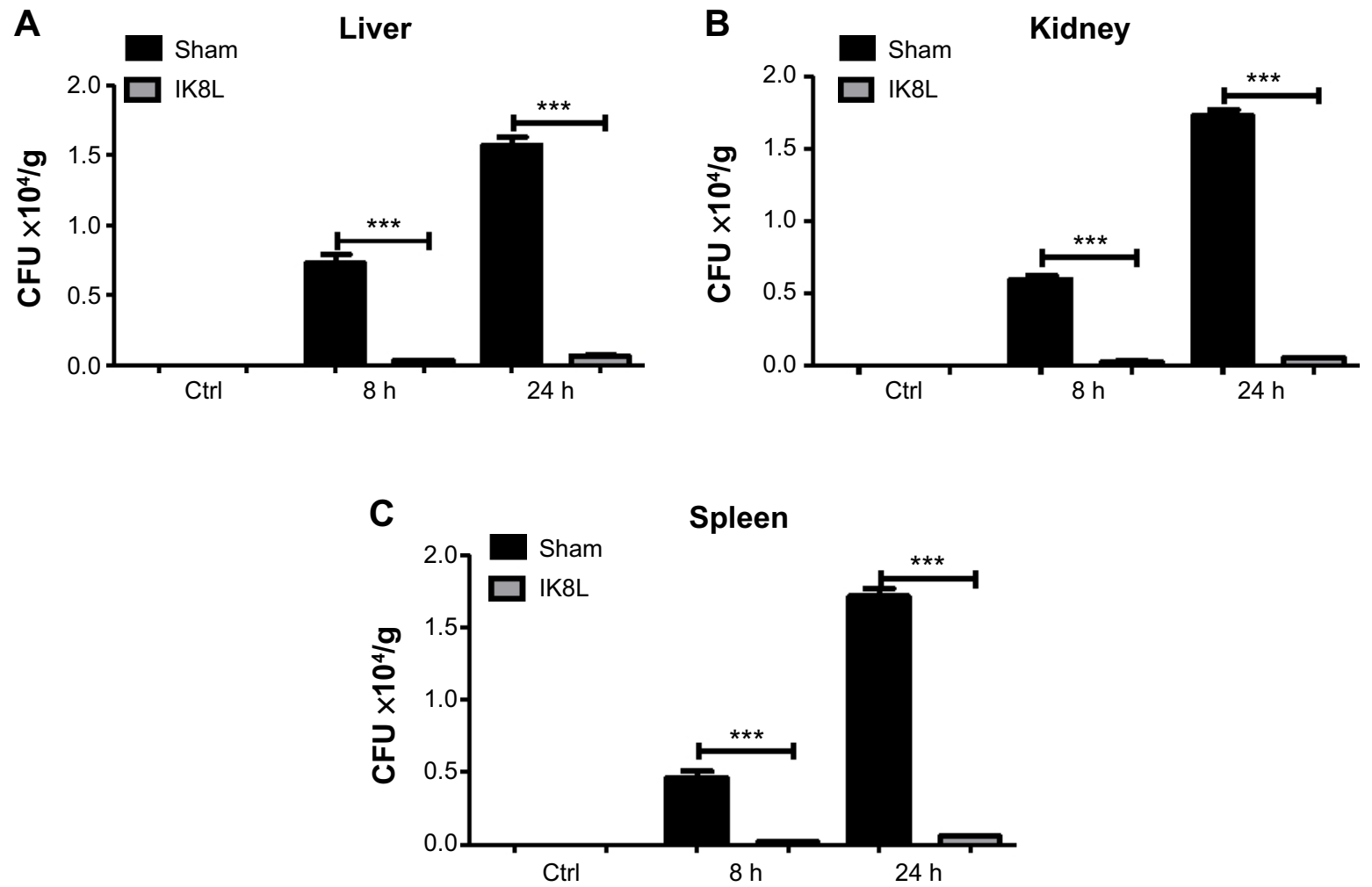

Figure SI IK8L decreased the dissemination of infection.

Notes: $(\mathbf{A}-\mathbf{C})$ The liver, spleen, and kidneys showed significantly decreased bacterial burdens after infection with Kp in IK8L-treated mice compared with sham-treated mice. IK8L-treated mice and sham-treated mice were infected with $I \times 10^{5} \mathrm{CFU} /$ mouse $K p$ at 8 and 24 hours. Fresh tissues were homogenized in PBS. The same amounts of tissue were evaluated for testing bacterial colonies and the unit is CFU/g. The data are representative of four mice per group. $* * * P<0.00 I ; M a n n-W$ hitney $U$-test.

Abbreviations: CFU, colony-forming unit; Ctrl, control; h, hours; Kp, Klebsiella pneumoniae; PBS, phosphate-buffered saline. 

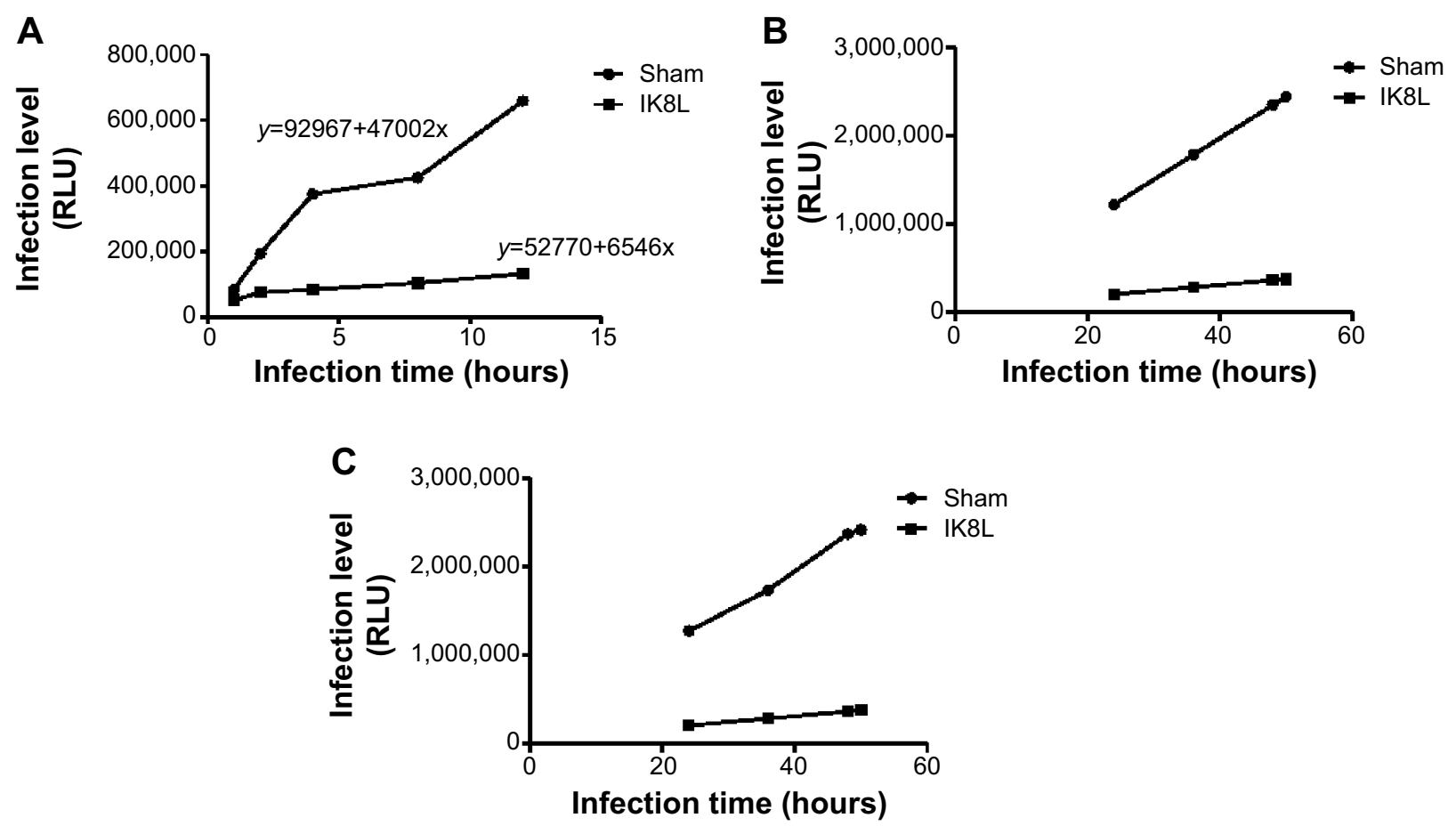

Figure S2 Prediction of infection with time using the mathematical model.

Notes: (A) Data obtained from in vivo imaging were quantified using relative LUC units of the two groups in sham-treated and IK8L-treated mice at I hour, 2 hours, 4 hours, 8 hours, and 12 hours. Sham- and IK8L-treated mice were infected with $1 \times 10^{5} \mathrm{CFU} /$ mouse of $K p$ Xen- 39 by nasal cavity. Semiquantitatively, bioluminescence intensity was obtained using IVIS XRII software. Regression equation was calculated using SPSS software calculations. (B) The data showing predicted relative LUC units of two groups according to two regression equations in (A) at 24 hours, 36 hours, 48 hours, and 50 hours according to our regression equation. (C) The data showing actual LUC units of the two groups in sham-treated and IK8L-treated mice at 24 hours, 36 hours, 48 hours, and 50 hours. Sham- and IK8L-treated mice were infected with I $\times 10^{5}$ CFU of Kp Xen-39 by nasal cavity.

Abbreviations: CFU, colony-forming units; LUC, luminescence unit counts.

International Journal of Nanomedicine

Dovepress

\section{Publish your work in this journal}

The International Journal of Nanomedicine is an international, peerreviewed journal focusing on the application of nanotechnology in diagnostics, therapeutics, and drug delivery systems throughou the biomedical field. This journal is indexed on PubMed Central, MedLine, CAS, SciSearch ${ }^{\circledR}$, Current Contents ${ }^{\circledR} /$ Clinical Medicine,
Journal Citation Reports/Science Edition, EMBase, Scopus and the Elsevier Bibliographic databases. The manuscript management system is completely online and includes a very quick and fair peer-review system, which is all easy to use. Visit http://www.dovepress.com/ testimonials.php to read real quotes from published authors. 